Björk et al. Supporting Information

\title{
Supporting Information: Single molecular imaging and spectroscopy of conjugated polyelectrolytes decorated on stretched aligned DNA
}

Fabrication of PDMS stamps. To fabricate the PDMS stamps, a mixture of 10:1 PDMS Sylgard Silcone Elastomer 184 and Sylgard Curing Agent 184 (Dow Corning Corporation, Midland, MI, USA) has been thoroughly mixed and degassed in a vacuum chamber. The solution has been casted onto a hydrophobic silicon wafer and cured for 1 hour in $80{ }^{\circ} \mathrm{C}$. The PDMS was left a couple of minutes to cool down and then carefully peeled of and cut into appropriate sizes.

Control experiments for $\lambda$-DNA stretching. Figure $S 1$ shows stretching of a $1 \mu \mathrm{g} / \mathrm{ml}$ solution of $\lambda$ DNA prestained with YOYO-1 (Molecular Probes) in 50 mM 2-Morpholinoethanesulfonic acid (MES) pH 5.5 buffer. The substrate is a glass slide where the surface has been modified using a PDMS stamp.

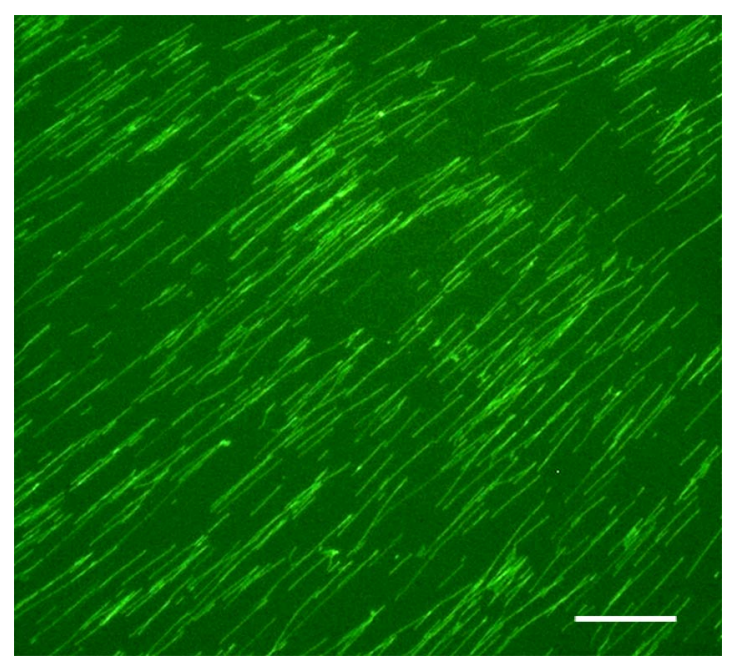

Figure S1. Image of stretched $\lambda$-DNA prestained with YOYO-1 on PDMS modified glass slide. Scale bar: $10 \mu \mathrm{m}$. 
As a control experiments for conjugation of polyelectrolyte after stretching of DNA on a surface we stretched a $1 \mu \mathrm{g} / \mathrm{ml}$ solution of $\lambda$-DNA prestained with YOYO-1 in $50 \mathrm{mM}$ MES pH 5.5 buffer. After the stretching of the prestained DNA, a $5 \mu \mathrm{g} / \mathrm{ml}$ POMT solution (MilliQ water) was used to wash parts of the surface with stretched DNA. Figure S2 show a border of a washed and an unwashed part of the stretched DNA.

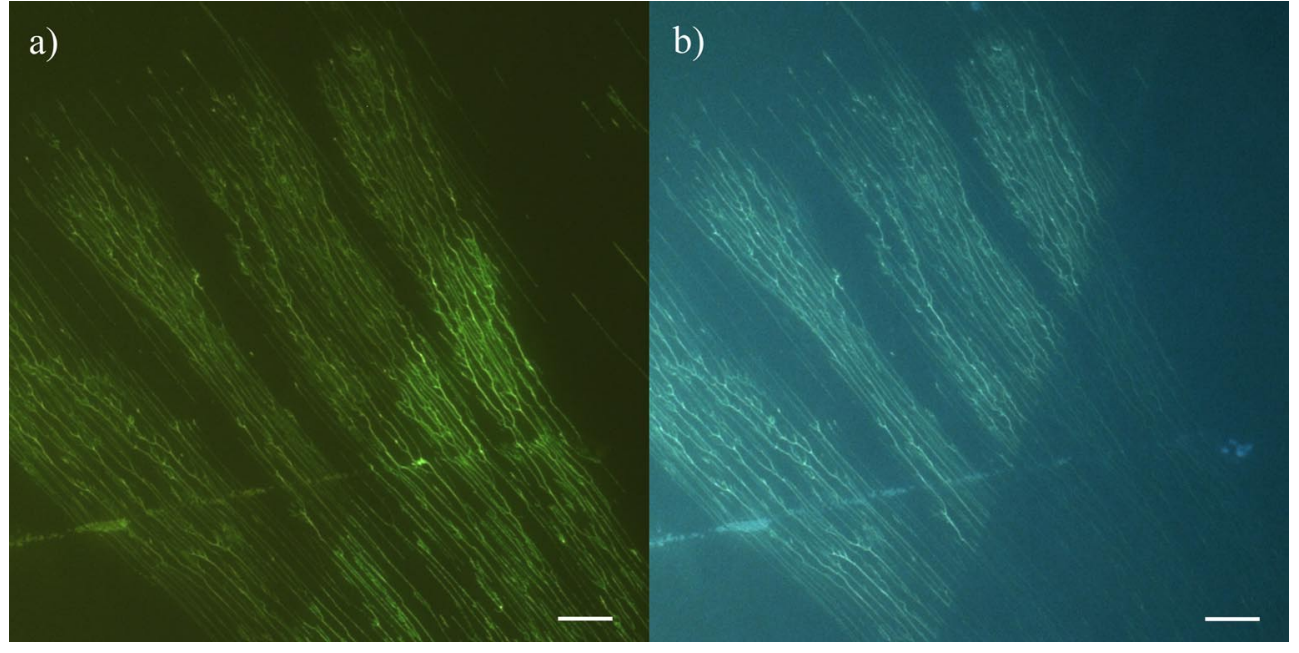

Figure S2. Fluorescence images of stretch DNA pre-stained with YOYO-1 on PMMA substrate, where parts of the stretched DNA have been washed with a POMT solution. (a) Excitation at $470 \mathrm{~nm}$, both POMT and YOYO-1 are excited. The bright part to the right show the fluorescence from YOYO-1, while the less bright part to the left show the fluorescence from POMT. (b) Excitation at $405 \mathrm{~nm}$, almost only POMT is excited. The bright part to the left show fluorescence from the POMT, while the less bright part to the right show fluorescence from YOYO-1. Scale bars: $10 \mu \mathrm{m}$.

Fluorescence spectroscopy. A stock solution containing $20 \mu \mathrm{M} \quad \lambda$-DNA in $50 \quad \mathrm{mM}$ Morpholinoethanesulfonic acid (MES) pH 5.5 buffer and stock solutions of $0.5 \mathrm{mg} / \mathrm{ml}$ POMT and POWT in the same buffer were prepared and incubated for $30 \mathrm{~min} .10 \mu \mathrm{l}$ of the polyelectrolyte solution was diluted to $350 \mu \mathrm{l}$ and mixed with $350 \mu \mathrm{l}$ MES buffer or $350 \mu \mathrm{l} \lambda$-DNA solution. The samples were 
incubated for $5 \mathrm{~min}$. The emission spectra were recorded at $400 \mathrm{~nm}$ excitation with an ISA Jobin-Yvon spex FluoroMax-3 spectrometer.

Single Molecular Spectroscopy. A home built wide-field fluorescence microscope based on commercial Olympus IX-71 inverted microscope was used to study fluorescence of single complexes. Fluorescence was excited using the $458 \mathrm{~nm}$ line of a cw argon-ion laser. The excitation density was ca. $80 \mathrm{~W} / \mathrm{cm}^{2}$ at the center of the irradiated spot. Fluorescence light was collected by an objective lens (Olympus LUCPlanFl 40x, NA 0.6) and projected onto a CCD camera (Photometrics, Cascade 512B). During all experiments the sample was kept in a home-build vacuum chamber $\left(10^{-2}\right.$ torr $)$ to increase photostability

Imaging of the complexes and recording of their fluorescence spectra were carried out simultaneously by using holographic grating (Edmund Optics, 70 lines/mm) placed in front of the CCD camera. The dispersion was horizontal. The distance between the CCD chip and the grating was such that zero-order and first-order of the diffraction appeared on the CCD chip at the same time. The zero-order gave an image and the first order gave a spectrum. Selection of a particular place on the sample was done by vertical slit with adjustable width placed in an intermediate image plane. Fluorescence spectra were corrected on transmission of the dichroic mirror and the excitation laser cut-off filter placed in the detection path. No corrections on the spectral sensitivity of the CCD camera were applied. Exposure time was up to 20 seconds depending on the brightness of the complexes. Instrumental response function has $\mathrm{FWHM} \approx 8 \mathrm{~nm}$

Figure S3 shows a time series for a stretched blinking CPE/DNA complex. The concentration of CPE is diluted around 10 times to $0.13 \mu \mathrm{g} / \mathrm{ml}$, while the DNA concentration is still $5 \mu \mathrm{g} / \mathrm{ml}$.

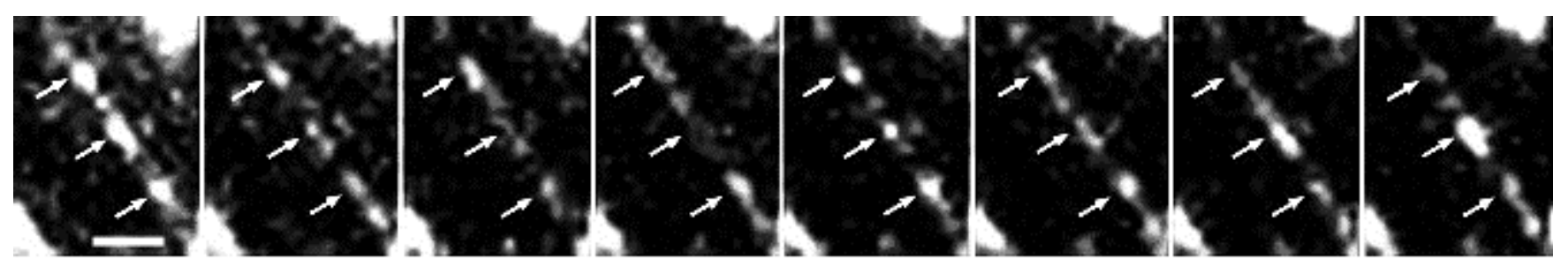


Figure S3. Time series of fluorescence images taken with an interval of around $1.5 \mathrm{~s}$ and $0.3 \mathrm{~s}$ exposure time for diluted concentration of POMT relative to that of DNA. Blinking spots are observed and marked with arrows. Scale bar: $2 \mu \mathrm{m}$ 